\title{
Machine-learning-based COVID-19 mortality prediction model and identification of patients at low and high risk of dying
}

\author{
Mohammad M. Banoei ${ }^{1,2}$, Roshan Dinparastisaleh ${ }^{3}$, Ali Vaeli Zadeh ${ }^{4}$ and Mehdi Mirsaeidi ${ }^{*}$ (D)
}

\begin{abstract}
Background: The coronavirus disease 2019 (COVID-19) pandemic caused by the SARS-Cov2 virus has become the greatest health and controversial issue for worldwide nations. It is associated with different clinical manifestations and a high mortality rate. Predicting mortality and identifying outcome predictors are crucial for COVID patients who are critically ill. Multivariate and machine learning methods may be used for developing prediction models and reduce the complexity of clinical phenotypes.

Methods: Multivariate predictive analysis was applied to 108 out of 250 clinical features, comorbidities, and blood markers captured at the admission time from a hospitalized cohort of patients $(N=250)$ with COVID-19. Inspired modification of partial least square (SIMPLS)-based model was developed to predict hospital mortality. Prediction accuracy was randomly assigned to training and validation sets. Predictive partition analysis was performed to obtain cutting value for either continuous or categorical variables. Latent class analysis (LCA) was carried to cluster the patients with COVID-19 to identify low- and high-risk patients. Principal component analysis and LCA were used to find a subgroup of survivors that tends to die.

Results: SIMPLS-based model was able to predict hospital mortality in patients with COVID-19 with moderate predictive power $\left(Q^{2}=0.24\right)$ and high accuracy $(A \cup C>0.85)$ through separating non-survivors from survivors developed using training and validation sets. This model was obtained by the 18 clinical and comorbidities predictors and 3 blood biochemical markers. Coronary artery disease, diabetes, Altered Mental Status, age $>65$, and dementia were the topmost differentiating mortality predictors. CRP, prothrombin, and lactate were the most differentiating biochemical markers in the mortality prediction model. Clustering analysis identified high- and low-risk patients among COVID-19 survivors.
\end{abstract}

Conclusions: An accurate COVID-19 mortality prediction model among hospitalized patients based on the clinical features and comorbidities may play a beneficial role in the clinical setting to better management of patients with COVID-19. The current study revealed the application of machine-learning-based approaches to predict hospital mortality in patients with COVID-19 and identification of most important predictors from clinical, comorbidities and blood biochemical variables as well as recognizing high- and low-risk COVID-19 survivors.

Keywords: COVID-19, Prediction model, Machine learning, SARS-CoV-2, Mortality

*Correspondence: msm249@med.miami.edu

${ }^{5}$ Division of Pulmonary and Critical Care, Department of Medicine, University of Miami, Miami, FL, USA

Full list of author information is available at the end of the article

\section{Background}

The COVID-19 disease has resulted in a substantial cause of morbidity and mortality across the world [1]. COVID19 disease presents with a wide range of clinical features original author(s) and the source, provide a link to the Creative Commons licence, and indicate if changes were made. The images or other third party material in this article are included in the article's Creative Commons licence, unless indicated otherwise in a credit line to the material. If material is not included in the article's Creative Commons licence and your intended use is not permitted by statutory regulation or exceeds the permitted use, you will need to obtain permission directly from the copyright holder. To view a copy of this licence, visit http://creativecommons.org/licenses/by/4.0/. The Creative Commons Public Domain Dedication waiver (http://creativeco mmons.org/publicdomain/zero/1.0/) applies to the data made available in this article, unless otherwise stated in a credit line to the data. 
spanning from no symptoms to multi-organ failure [2]. Although SARS-CoV-2 mainly affects the lungs and is associated with developed acute respiratory distress syndrome (ARDS), it can impact cardiovascular, neurological, renal, and vascular complications associated with high mortality [3]. The precise prognostication of COVID-19 clinical outcome is more challenging due to the high variability in disease severity that could essentially be helpful for effective triage and efficient allocation of limited resources (i.e., beds, ventilators). More accurate subclassification of COVID-19 is essential for prognostication and identification of severity [4].

It has been shown that the pathological, physiological, and immunological responses do not sufficiently discriminate patients with non-severe and severe form due to the high level of complexity of these features [4]. A combination of clinical features and biochemical markers has been studied to identify the clinical subtype of COVID19. Data mining and machine learning (ML) approach could potentially be applied to such diverse multimodal data for the classification of patients with COVID-19 [4]. Therefore, AI has been used for the diagnosis of COVID19 pneumonia, stratification of patients and developing a prediction model of patterns of spread [5]. AI- and MLbased approach can be used as either diagnostic tool or a prognostic model to predict outcome [6]. Many studies have characterized the association of major risk factors with the COVID mortality such as higher age, cardiovascular disease, chronic respiratory disease, diabetes, hypertension, smoking history, and obesity [7]. However, they could not be strong individual predictors mainly through using conventional statistical analysis due to high degree of complexity and collinearity among the data.

In the present study, we aimed to apply ML-based algorithms to generate a mortality prediction model for hospitalized COVID-19 patients as well as classification of patients to verify the low- and high-risk groups.

\section{Methods and materials Data collection}

In a retrospective study, we used clinical data from 400 patients with a polymerase chain reaction (PCR) test confirmed patients with COVID-19. Data were collected from patients admitted at the University of Miami Hospital, Miller School of Medicine, Miami, FL, USA, since June 2020. A total of 250 variables including biochemical and clinical data were collected at various times (hospital admission, ICU admission, hospital discharge). The admission time data were considered as the data at presentation. These data including demographic variables in addition to comorbidities, patients' vitals, anthropometric measurements, chronic treatments, and laboratory works were obtained from the patient's electronic records. In the processing dataset, the missing values level of each variable were found among the current cohort. The maximum level of missing values was $7 \%$ among the variables. Using imputation methods, new data were created by replacing all missing values with the estimated values using mean imputation. Continuous variables were median fold normalized, log-transformed, and univariance scaled before statistical analysis.

\section{Definitions of variables}

Table 1 summarizes patients' demographics, clinical variables, comorbidities, and their association with hospital mortality and survival of patients with COVID-19.

In this table, the patient's level of consciousness, when it was available, is shown based on Glasgow Coma Scale (GCS). We mentioned the patient's temperature in Fahrenheit. Respiratory rate (RR) indicates the number of breaths per minute, and the heart rate (HR) demonstrates the number of heart beats per minute. The patients' systolic and diastolic blood pressure (BP) is presented in millimeters of mercury. The percentage of oxygen-saturated hemoglobin to the total hemoglobin is displayed by $\mathrm{O}_{2}$ saturation, and $\mathrm{ynO}_{2}$ shows whether the patient was on oxygen during the hospitalization. The percentage of the oxygen that the patient inhales is presented by $\mathrm{FiO}_{2}$ (the fraction of inspired oxygen). $\mathrm{O}_{2}$ flow (lpm) indicates the required oxygen flow in liters per minute. Nursing home shows whether the patient was in a nursing home or long-term care facility before hospitalization. Patient delay $\geq 7$ is used to define patients who delayed at least seven days to seek medical assistance after the onset of symptoms.

Smoking and alcohol are used to show the patient's history of exposure to these toxins. The patient's vaccination status against influenza (flu vaccine) and pneumonia (pneumonia vaccine) is included as per medical records or informed by the patient at the time of inclusion in the study.

Altered Mental Status (AMS) refers to any decline in the patient's mental capacity noted through the physical exam. The loss of sense of smell and taste is displayed as anosmia and ageusia. We collected data related to the use of any chronic treatments or chemotherapy. Home O2 shows whether the patient was on supplemental oxygen therapy at home. We have also determined whether the patients are on local (inhaled steroids) or systemic corticosteroids (prednisone). ACE inhibitors indicate that the patient was on chronic treatment with angiotensinconverting enzyme inhibitors, and ARBs refer to the chronic use of the angiotensin 11 receptor blockers. To evaluate the predictive value of imaging tests, we have collected data about radiological findings in the patient's 
Table 1 Distribution of patients' demographics, clinical variables, and comorbidities between hospital mortality and survival of patients with COVID-19

\begin{tabular}{|c|c|c|c|c|}
\hline & \multirow[t]{2}{*}{ Variables } & \multicolumn{2}{|c|}{ Hospitalized death } & \multirow[t]{2}{*}{$P$ value } \\
\hline & & Yes & No & \\
\hline 1 & Male & $22(70.9)$ & $118(53.8)$ & 0.085 \\
\hline 2 & Age (years) $M \pm S D$ & $78.1 \pm 10.6$ & $60.58 \pm 16.78$ & $<0.0001^{*}$ \\
\hline 3 & Height $(\mathrm{cm}) \mathrm{M} \pm \mathrm{SD}$ & $161.19 \pm 31.69$ & $167.04 \pm 15.89$ & 0.106 \\
\hline 4 & Weight (lb.) M $\pm S D$ & $176.93 \pm 25.77$ & $180.37 \pm 47.41$ & 0.467 \\
\hline 5 & $\mathrm{GCS} M \pm S D$ & $13.50 \pm 3.89$ & $14.80 \pm 1.50$ & $0.009^{*}$ \\
\hline 6 & Temperature $\mathrm{M} \pm \mathrm{SD}$ & $100.04 \pm 1.41$ & $99.31 \pm 6.37$ & 0.528 \\
\hline 7 & Respiratory rate (RR) & $27.68 \pm 15.23$ & $22.12 \pm 6.2$ & $<0.0001^{*}$ \\
\hline 8 & Heart rate $M \pm S D$ & $95.52 \pm 25.44$ & $93.34 \pm 20.91$ & 0.599 \\
\hline 9 & Blood pressure (systolic) $\mathrm{M} \pm \mathrm{SD}$ & $123.58 \pm 30.77$ & $130.54 \pm 24.15$ & 0.149 \\
\hline 10 & Blood pressure (diastolic) $\mathrm{M} \pm \mathrm{SD}$ & $67.58 \pm 19.64$ & $74.63 \pm 16.68$ & 0.032 \\
\hline 11 & $\mathrm{O}_{2}$ saturation $\mathrm{M} \pm \mathrm{SD}$ & $93.63 \pm 5.81$ & $93.35 \pm 5.57$ & 0.118 \\
\hline 12 & $\mathrm{ynO}_{2} \mathrm{M} \pm \mathrm{SD}$ & $0.76 \pm 0.43$ & $0.56 \pm 0.49$ & 0.058 \\
\hline 13 & $\mathrm{FiO}_{2} \% \mathrm{M} \pm \mathrm{SD}$ & $81.29 \pm 29.71$ & $50.22 \pm 59.81$ & $0.042^{*}$ \\
\hline 14 & $\mathrm{O}_{2}$ flow (lpm) $\mathrm{M} \pm \mathrm{SD}$ & $23.07 \pm 21.79$ & $8.55 \pm 14.0$ & $0.002^{*}$ \\
\hline 15 & Nursing home & $12(38.7)$ & $26(11.9)$ & $0.001^{*}$ \\
\hline 16 & H1N1 & $21(67.7)$ & $135(61.6)$ & 0.295 \\
\hline 17 & European American & $21(67.7)$ & $126(57.5)$ & 0.332 \\
\hline 18 & Hispanic & $153(67.4)$ & $86(58.1)$ & 0.068 \\
\hline 19 & African-American & $4(12.9)$ & $57(26.02)$ & 0.074 \\
\hline 20 & Shelter/homeless & 0 & $7(3.19)$ & 0.602 \\
\hline 21 & Asian & $1(3.2)$ & $3(1.36)$ & 0.413 \\
\hline 22 & $>$ one race & $2(6.4)$ & $18(8.21)$ & 1.00 \\
\hline 23 & Patient delay $\geq 7$ & $6(19.35)$ & $54(24.65)$ & 0.788 \\
\hline 24 & Smoking & $1(3.2)$ & $16(7.3)$ & 0.702 \\
\hline 25 & Alcohol & $4(12.9)$ & $62(28.31)$ & 0.166 \\
\hline 26 & Flu vaccine & $4(12.9)$ & $46(21)$ & 1.00 \\
\hline 27 & Pneumonia vaccine & $5(16.12)$ & $41(18.72)$ & 0.491 \\
\hline 28 & Cough & $19(61.2)$ & $126(57.5)$ & 0.300 \\
\hline 29 & Sore throat & $2(6.4)$ & $6(2.73)$ & 0.207 \\
\hline 30 & Rhinorrhea & $1(3.2)$ & $9(4.10)$ & 1.000 \\
\hline 31 & Sputum & $2(6.4)$ & $21(9.58)$ & 1.000 \\
\hline 32 & Chest pain & 0 & $34(15.52)$ & $0.031^{*}$ \\
\hline 33 & Dyspnea & $24(77.41)$ & $132(60.27)$ & 0.067 \\
\hline 34 & Hemoptysis & 0 & $4(1.82)$ & 1.000 \\
\hline 35 & Fever & $19(61.2)$ & $136(62.1)$ & 0.063 \\
\hline 36 & Chills & $6(19.35)$ & $66(30.13)$ & 0.500 \\
\hline 37 & Headache & 0 & $21(9.58)$ & 0.140 \\
\hline 38 & Myalgia & $6(19.35)$ & $54(24.65)$ & 0.816 \\
\hline 39 & Abdominal pain & $2(6.4)$ & $31(14.15)$ & 0.545 \\
\hline 40 & Diarrhea & $4(12.9)$ & $40(18.26)$ & 0.793 \\
\hline 41 & Nausea-vomiting & $4(12.9)$ & $34(15.52)$ & 1.000 \\
\hline 42 & Altered Mental Status (AMS) & $10(32.25)$ & $19(8.67)$ & $<0.0001^{*}$ \\
\hline 43 & Anosmia (loss of smell) & 0 & $6(2.73)$ & 1.000 \\
\hline 44 & Ageusia (loss of taste) & 0 & $3(1.36)$ & 1.000 \\
\hline 45 & Chronic treatment & $23(74.19)$ & $124(56.62)$ & 0.072 \\
\hline 46 & On any chemotherapy & $3(9.67)$ & $15(6.84)$ & 0.464 \\
\hline 47 & Home $\mathrm{O}_{2}$ & $5(16.12)$ & $6(2.73)$ & $0.006^{*}$ \\
\hline
\end{tabular}


Table 1 (continued)

\begin{tabular}{|c|c|c|c|c|}
\hline & Variables & Hospitaliz & & $P$ value \\
\hline & & Yes & No & \\
\hline 48 & Inhaled steroid & $3(9.67)$ & $23(10.50)$ & 1.000 \\
\hline 49 & Prednisone & $3(9.67)$ & $14(6.39)$ & 0.442 \\
\hline 50 & ACE inhibitors & $9(29.03)$ & $32(14.61)$ & 0.061 \\
\hline 51 & ARBs & $6(19.35)$ & $27(12.32)$ & 0.253 \\
\hline 52 & Statins & $12(38.7)$ & $65(29.6)$ & 0.290 \\
\hline 53 & Prior ER visit (on past 12 months) & $10(32.25)$ & $81(36.98)$ & 1.000 \\
\hline 54 & Any prior hospitalization & $12(38.7)$ & $83(37.89)$ & 0.378 \\
\hline 55 & Consolidation on the imaging & $13(41.93)$ & $34(15.52)$ & $0.002^{*}$ \\
\hline 56 & Pleural effusion on the imaging & $6(19.35)$ & $25(11.41)$ & 0.250 \\
\hline 57 & Pulmonary infiltrates on the imaging & $17(54.83)$ & $103(47.03)$ & 0.568 \\
\hline 58 & Asthma & $2(6.4)$ & $28(12.78)$ & 0.548 \\
\hline 59 & Pulmonary embolism (PE) & 0 & $8(3.65)$ & 0.601 \\
\hline 60 & COPD & $4(12.9)$ & $16(7.30)$ & 0.273 \\
\hline 61 & Emphysema & $1(3.2)$ & $4(1.82)$ & 0.486 \\
\hline 62 & Bronchiectasis & 0 & $2(0.91)$ & 1.000 \\
\hline 63 & $\mathrm{CHF}$ & $6(19.35)$ & $8(3.65)$ & $0.003^{*}$ \\
\hline 64 & CAD & $11(35.48)$ & 14 (6.39) & $<0.0001^{*}$ \\
\hline 65 & $\mathrm{AMI}$ & $6(19.35)$ & $3(1.36)$ & $<0.0001^{*}$ \\
\hline 66 & AFib & $3(9.67)$ & $19(8.67)$ & 0.740 \\
\hline 67 & Hypertension & $26(83.87)$ & $126(57.53)$ & $0.002^{*}$ \\
\hline 68 & Peripheral vascular diseases & $2(6.4)$ & $12(5.47)$ & 0.676 \\
\hline 69 & Stroke & $4(12.9)$ & 14 (6.39) & 0.138 \\
\hline 70 & Dementia & $8(25.08)$ & $17(7.76)$ & $0.004^{*}$ \\
\hline 71 & Chronic renal failure (CRF) & $6(19.35)$ & $21(9.58)$ & 0.107 \\
\hline 72 & Hemodialysis & $3(9.67)$ & $6(2.73)$ & 0.079 \\
\hline 73 & Liver diseases & 0 & $8(3.65)$ & 0.601 \\
\hline 74 & Diabetes & $21(67.7)$ & $59(26.94)$ & $<0.0001^{*}$ \\
\hline 75 & Peptic ulcer disease (PUD) & $1(3.2)$ & $11(5.02)$ & 1.000 \\
\hline 76 & Leukemia & $1(3.2)$ & $4(1.82)$ & 0.473 \\
\hline 77 & Lymphoma & $1(3.2)$ & $7(3.19)$ & 1.000 \\
\hline
\end{tabular}

chest X-ray. Consolidation on the imaging refers to the existence of dense material in the alveoli and small airways. The presence of excess fluid accumulation in pleural space is listed as pleural effusion on the imaging, and the existence of dense material in the interstitium is mentioned as pulmonary infiltrates on the imaging.

The chronic health conditions of participants were collected to determine the impact of comorbidities on the outcome. These conditions include diabetes, chronic obstructive pulmonary disease (COPD), emphysema, pulmonary embolism (PE), bronchiectasis, interstitial lung disease (ILD), congestive heart failure (CHF), coronary artery disease (CAD), acute myocardial infarction (AMI), atrial fibrillation (AFib), hypertension, peripheral vascular disease, stroke, dementia, any stage of chronic renal failure (CRF), liver disease, peptic ulcer disease
(PUD), connective tissue disorder, leukemia, lymphoma, dependence on hemodialysis, and asthma.

\section{Statistical analysis}

To establish a prediction model, we used the statistically inspired modification of partial least square (SIMPLS) analysis for the clinical data and blood markers collected at admission time. SIMPLS, an algorithm of PLS (a linear machine learning method) $[8,9]$, was carried out with two training and validation sets. To develop the best prediction model, SIMPLS-based prediction model was built using all variables as primary model. SIMPLS predicts the outcome response to variables by fitting a regression model $(Y=X B)$ that is derived using the variables. Since all variables were not important to predict outcome, secondly variable reduction in SIMPLS was done to characterize useful predictor in explaining variation 
Table 2 Distribution of patients' laboratory variables between hospital mortality and survival of patients with COVID-19

\begin{tabular}{|c|c|c|c|c|}
\hline \multirow[t]{2}{*}{ Variables } & \multicolumn{2}{|c|}{ Hospitalized death } & \multirow[t]{2}{*}{$P$ value } & \multirow[t]{2}{*}{ Normal range } \\
\hline & Yes & No & & \\
\hline Leukocytes $\left(10^{3} / \mu \mathrm{L}\right)$ & $11.75 \pm 7.69$ & $7.73 \pm 4.55$ & $<0.0001^{*}$ & $4.5-11$ \\
\hline Neutrophils $\left(10^{3} / \mu \mathrm{L}\right)$ & $13.34 \pm 14.92$ & $8.59 \pm 13.59$ & 0.074 & $2.5-6$ \\
\hline Lymphocytes $\left(10^{3} / \mu \mathrm{L}\right)$ & $2.32 \pm 5.16$ & $8.59 \pm 13.59$ & 0.577 & $1-4$ \\
\hline Eosinophil $\left(10^{3} / \mu \mathrm{L}\right)$ & $0.35 \pm 1.38$ & $0.07 \pm 0.18$ & $0.006^{*}$ & $0.05-0.3$ \\
\hline Hemoglobin (g/dL) & $12 \pm .2 .36$ & $12.81 \pm 9.23$ & 0.627 & $13.5-17.5$ \\
\hline Hematocrit (\%) & $37.24 \pm 7.47$ & $37.38 \pm 7.34$ & 0.921 & $36-50$ \\
\hline Platelets $\left(10^{3} / \mu \mathrm{L}\right)$ & $210 \pm 138.88$ & $227.40 \pm 110.23$ & 0.428 & $200-500$ \\
\hline $\operatorname{ESR}(\mathrm{mm} / \mathrm{hr})$ & $47.75 \pm .35 .47$ & $45.31 \pm 29.39$ & 0.801 & $0-29$ \\
\hline BUN (mg/dL) & $39.36 \pm 22.97$ & $21.24 \pm 19.55$ & $<0.0001^{*}$ & $6-24$ \\
\hline Creatinine $(\mathrm{mg} / \mathrm{dL})$ & $2.23 \pm 2.08$ & $1.59 \pm 2.20$ & 0.129 & $0.74-1.35$ \\
\hline $\mathrm{Na}(\mathrm{mEq} / \mathrm{L})$ & $139.81 \pm 9.02$ & $137.05 \pm 6.06$ & $0.028^{*}$ & $135-145$ \\
\hline $\mathrm{K}(\mathrm{mmol} / \mathrm{L})$ & $4.57 \pm 1.32$ & $4.21 \pm 0.66$ & $0.017^{*}$ & $3.6-5.2$ \\
\hline Ferritin (ng/mL) & $2292 \pm 3600$ & $1060 \pm 1742$ & $0.006^{*}$ & $20-250$ \\
\hline CRP (mg/dL) & $13.11 \pm 9.47$ & $10.85 \pm 11.50$ & 0.326 & $0.3-1.0$ \\
\hline $\mathrm{PCT}(\mathrm{ng} / \mathrm{mL})$ & $3.42 \pm 7.00$ & $3.44 \pm 18.46$ & 0.980 & $<0.5$ \\
\hline Lactate (mmol/L) & $48.84 \pm 172.95$ & $5.42 \pm 34.24$ & $0.003^{*}$ & $0.5-2.2$ \\
\hline Troponin (ng/mL) & $105.81 \pm 448.26$ & $0.02 \pm 0.03$ & $0.010^{*}$ & $<0.04$ \\
\hline CK (U/L) & $438.37 \pm 567.66$ & $242.80 \pm 452.35$ & 0.087 & 22-198 \\
\hline BNP $(\rho g / m L)$ & $4307.76 \pm 5997.9$ & $3098.26 \pm 3450.4$ & 0.635 & $<300$ \\
\hline $\mathrm{LDH}(\mathrm{U} / \mathrm{L})$ & $606.00 \pm 468.67$ & $393.26 \pm 224.11$ & $<0.0001^{*}$ & $140-280$ \\
\hline Fibrinogen (mg/dL) & $656.00 \pm 153.09$ & $538.83 \pm 165.68$ & 0.288 & $200-400$ \\
\hline $\operatorname{ALT}(\mathrm{U} / \mathrm{L})$ & $107.38 \pm 290.70$ & $55.41 \pm 85.80$ & $0.048^{*}$ & $7-55$ \\
\hline AST (U/L) & $258.66 \pm 983.07$ & $59.26 \pm 70.67$ & $0.005^{*}$ & $5-40$ \\
\hline Albumin (g/dL) & $3.19 \pm 0.77$ & $3.62 \pm 0.55$ & $<0.0001^{*}$ & $3.4-5.4$ \\
\hline D-dimer $(\mu \mathrm{g} / \mathrm{mL})$ & $5.35 \pm 6.25$ & $4.85 \pm 25.40$ & 0.936 & $0.05-6.5$ \\
\hline Bilirubin (mg/dL) & $0.56 \pm 0.33$ & $0.66 \pm 1.09$ & 0.625 & 0.3 \\
\hline Prothrombin (Second) & $15.70 \pm 2.76$ & $14.77 \pm 2.58$ & 0.160 & $11-13.5$ \\
\hline APTT (Second) & $59.53 \pm 48.96$ & $36.70 \pm 19.17$ & $<0.0001^{*}$ & $30-40$ \\
\hline $\mathrm{pH}$ & $7.34 \pm 0.11$ & $7.30 \pm 0.49$ & 0.684 & $7.35-4.45$ \\
\hline $\mathrm{PaCo} 2(\mathrm{~mm} \mathrm{Hg})$ & $36.28 \pm 17.08$ & $35.68 \pm 12.13$ & 0.841 & $38-42$ \\
\hline FiO2_lab & $75.20 \pm 29.90$ & $39.04 \pm 25.53$ & $<0.0001^{*}$ & \\
\hline Bicarbonate (mEq/L) & $21.18 \pm 4$ & $22.79 \pm 29.90$ & 0.140 & $23-30$ \\
\hline
\end{tabular}

in the predictor variable as well as their correlation to outcome. Variable reduction was applied to remove out the factors that were not useful in predicting outcome according to the variable important for the projection (VIP) value of each variable. VIP values were obtained through weighted sum of squares of the weights using SIMPLS analysis [10]. Thus, the contribution of variables in the SIMPLS models was assessed using VIP score. Based on the general agreement, the variables with the VIP values more than 1.0 were considered as important predictors [11]. The variables with lack of predictive ability $(\mathrm{VIP}<1.0)$ were removed from the basic prediction model.
The prediction model was created using the most differentiating clinical and biochemical variables (VIP > 1.0). The validation set automatically and randomly was created including $35 \%$ of out 250 hospitalized patients. In the absence of external validation cohort, splitting study cohort into training and validation sets is most known approach for internal validation of multivariate and machine-learning-based prediction mode.

SIMPLS was performed using the leave-one-out method of cross-validation $(\mathrm{CV})$. The $\mathrm{CV}$ method is also known as internal validation. SIMPLS analysis was assessed using $Q^{2}$, the goodness for predictability, and $R^{2} Y$, the goodness of variability. The best model was selected based on the number of factors for which $Q^{2}$ 
Table 3 Importance values (VIP) of 21 most differentiation among 108 variables used in the primary model

\begin{tabular}{lll}
\hline & Variables & VIP \\
\hline 1 & CAD & 2.1045 \\
2 & Diabetes & 1.9098 \\
3 & Age $>65$ & 1.7433 \\
4 & AMS & 1.6922 \\
5 & Dementia & 1.6309 \\
6 & Nursing home & 1.5545 \\
7 & Q saturation $<88$ & 1.5252 \\
8 & yno2 & 1.4903 \\
9 & Consolidation & 1.4654 \\
10 & Hypertension & 1.4226 \\
11 & Atrial fibrillation & 1.3789 \\
12 & Alcohol & 1.2563 \\
13 & Chest pain & 1.1566 \\
14 & Peripheral vascular disease & 1.1133 \\
15 & Prothrombin & 1.0855 \\
16 & Stroke & 1.0665 \\
17 & Headache & 1.0412 \\
18 & Dyspnea & 1.0212 \\
19 & CRP & 1.0125 \\
20 & Lactate & 1.0012 \\
21 & Smoking & 1.0011 \\
\hline & &
\end{tabular}

was larger and had not started decreasing with the highest $R^{2} Y$. The range of $R^{2}$ and $Q^{2}$ varies between 0 and 1 , the higher level showing higher predictive accuracy. Depending on data, the thresholds for the model performance change, generally $R^{2}$ greater than 0.67 and 0.33 , are considered as high and moderate predictive accuracy, respectively. Although $Q^{2}$ value greater than zero shows the model is predictive, $Q^{2}$ value with a range $0.2-0.4$ is considered as a model with moderate predictability. Close $R^{2}$ and $Q^{2}$ show a lack of overfitting and the SIMPLS model works independently of the specific data [12, 13].

The $Q^{2}$ and $R^{2} Y$ were computed using the training set and were verified using the validation set that make the model more realistic. Validation set was randomly selected from study cohort in a blinded approach.

Also, the partition analysis was used to creating a decision tree of the partition of data according to a relationship between the outcome and predictors. The data were partitioned into training and validation sets. The partition algorithm was to search all possible splits of predictors to best predict the response. The most differentiating clinical predictors obtained by SIMPLS were used for the partition analysis. AUC were obtained for both training and validation sets through the partition analysis based on the most important variables that were selected strong predictors in the SIMPLS-based prediction model.

We also used the partition analysis to obtain cutting value for either continuous or categorical (nominal or ordinal) variables such as age, heart rate, respiratory rate, and BMI. PCA and clustering were performed to identify subgroups particularly survivor subgroups. PCA was carried out in two steps. The first step was based on all variables to find outliers and trends and the step was using the most differentiating predictors obtained by SIMPLS. PCA and clustering were to help to find a subgroup of survivors that tends to hospital death. Latent class analysis (LCA) was carried to cluster the patients with COVID-19. Clustering was to help to identify the high-risk patients for dying. All paraclinical variables were normalized and transformed to use independently or in combination with clinical data for predicting hospital mortality.

\section{Results \\ Patients' characteristics}

A total of 250 hospitalized patients with RT-PCR confirmed COVID-19 enrolled in the study, and 31 (12.4\%) patients died in hospital. Table 1 shows the demographic characteristics, comorbidities, and outcomes of patients with COVID-19 that were admitted to MICU. The table shows, age, respiratory rate, $\mathrm{FiO}_{2} \%, \mathrm{O}_{2}$ flow (lpm), having been in nursing home, chest pain, Altered Mental Status (AMS), having been on home supplemental $\mathrm{O}_{2}$ therapy, pulmonary consolidation on the imaging, chronic heart failure $(\mathrm{CHF})$, coronary artery disease (CAD), acute myocardial infarction (AMI), dementia, hypertension, and diabetes mellitus were significantly different between the two cohorts. Table 2 shows the laboratory variables among survived and died patients.

\section{Predicting hospital mortality using clinical and paraclinical data}

The multivariate approach showed that patients' demographics, clinical variables, comorbidities, and biochemical markers can be used for predicting hospital mortality outcomes. SIMPLS analysis was carried using most differentiating variables (VIP > 1.0) [11] to establish the prediction model. The prediction model was developed on 172 patients in the training set and 78 patients in the validation set. Two-factor-based SIMPLS models had moderate predictability $\left(Q^{2}=0.24\right)$ with the variability of $R^{2}=0.37$ using a total of 21 variables that contributed to the prediction models. Table 3 also shows that CAD is the most important variable associated with mortality followed by diabetes mellitus, AMS, and age $>65$.

Further, the coefficient plot revealed that the age $>65$, nursing home, headache, dyspnea, AMS, consolidation, 


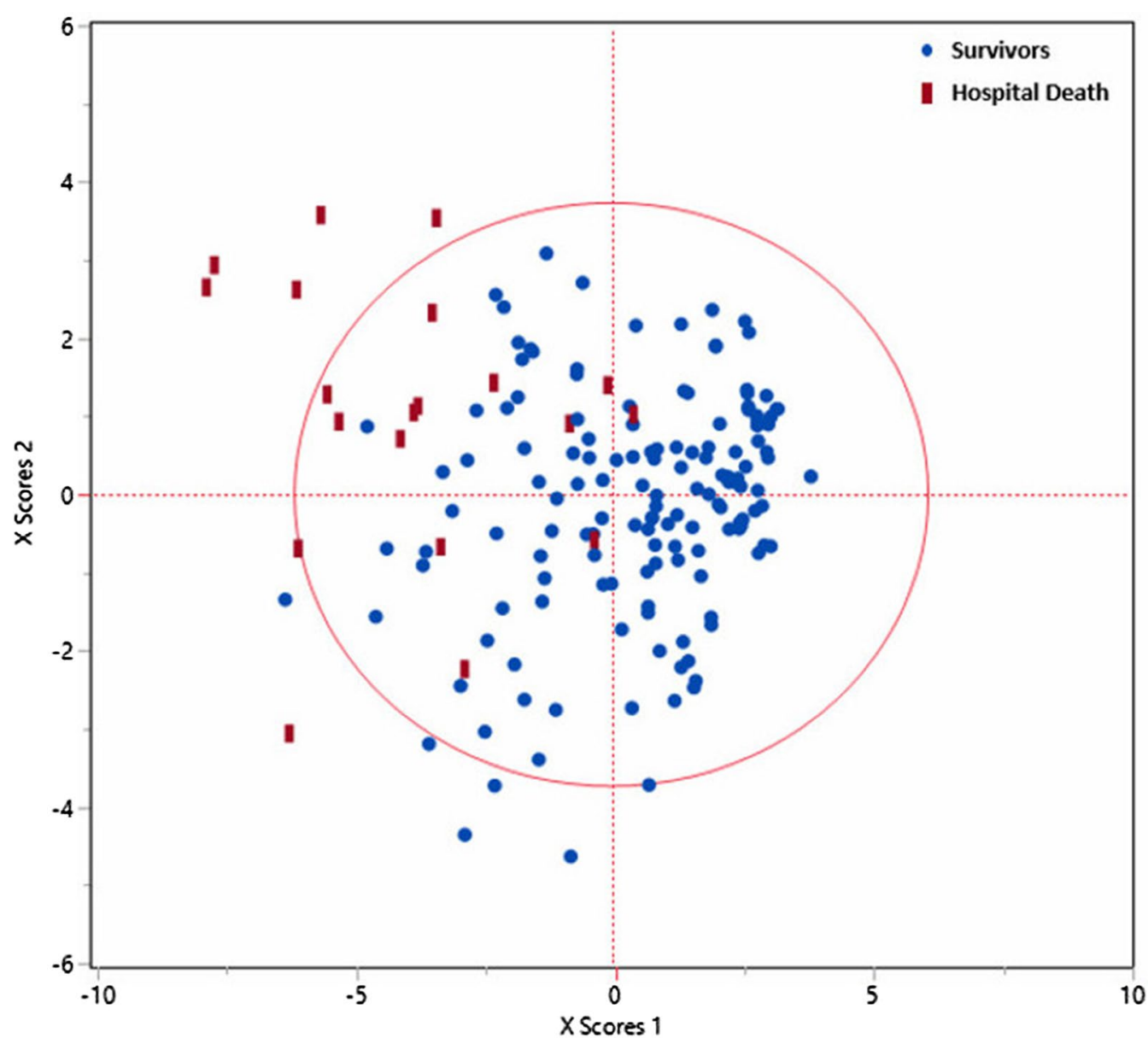

Fig. 1 SIMPLS-based scatter plot shows a good separation between hospital mortality of patients with COVID-19 from survivors. The figure illustrates only the training set-based scatter plot

O2 saturation $<88$, yno2, CAD, diabetes, alcohol, hypertension, stroke, dementia, prothrombin, and CRP were positively correlated with mortality among patients with COVID-19. On the other hand, chest pain, smoking, hypertension, atrial fibrillation, and peripheral vascular disease were negatively correlated with mortality. Scatterplot using two factors is characterized by adequately discriminating between patients who died and those who survived from COVID-19 in hospital ensuring accurate prediction of clinical variables (Fig. 1).

Further multivariate correlation analysis (Table 3) showed that CAD, diabetes, hypertension, AMS, dementia, stroke, atrial fibrillation, O2 saturation $<88$, yno2, nursing home, and age $>65$ are correlated together and mortality. Also, $\mathrm{O}^{2}$ saturation $<88$, lactate, dyspnea, consolidation in chest images, AMS, respiratory rate $>20$ and $\mathrm{yNO}^{2}$ were correlated together. Age $>65$, dementia, hypertension, and nursing home were closely intercorrelated. Also, the correlation analysis showed that alcohol and headache had a more negative correlation with most variables such as nursing home, diabetes, dementia, hypertension, CAD, and AMS. Only prothrombin and CRP were correlated only together, and lactate was correlated with $\mathrm{O} 2$ saturation $<88$, yno2 and atrial fibrillation (Table 3). Predictive partition analysis verified that the above-mentioned most differentiating clinical and blood maker variables are strong predictors to partition hospital mortality and survivors according to $\mathrm{AUC}=0.95$ and $\mathrm{AUC}=0.91$ for the training and validation sets, respectively (Fig. 2). The sensitivity, specificity, and accuracy were $80 \%, 92 \%$, and $90 \%$ for the training set and $75 \%$, $90 \%$, and $87 \%$ for the validation set, respectively.

Decision tree-based partition analysis revealed that age $<65$ and either absence or presence of diabetes were involved to partition at least $50 \%$ of survivors. Also, age $>65$, the $\mathrm{O} 2$ saturation condition, chest pain, and CAD had the highest portion for the partitioning of hospital death from survivors (Fig. 3). 

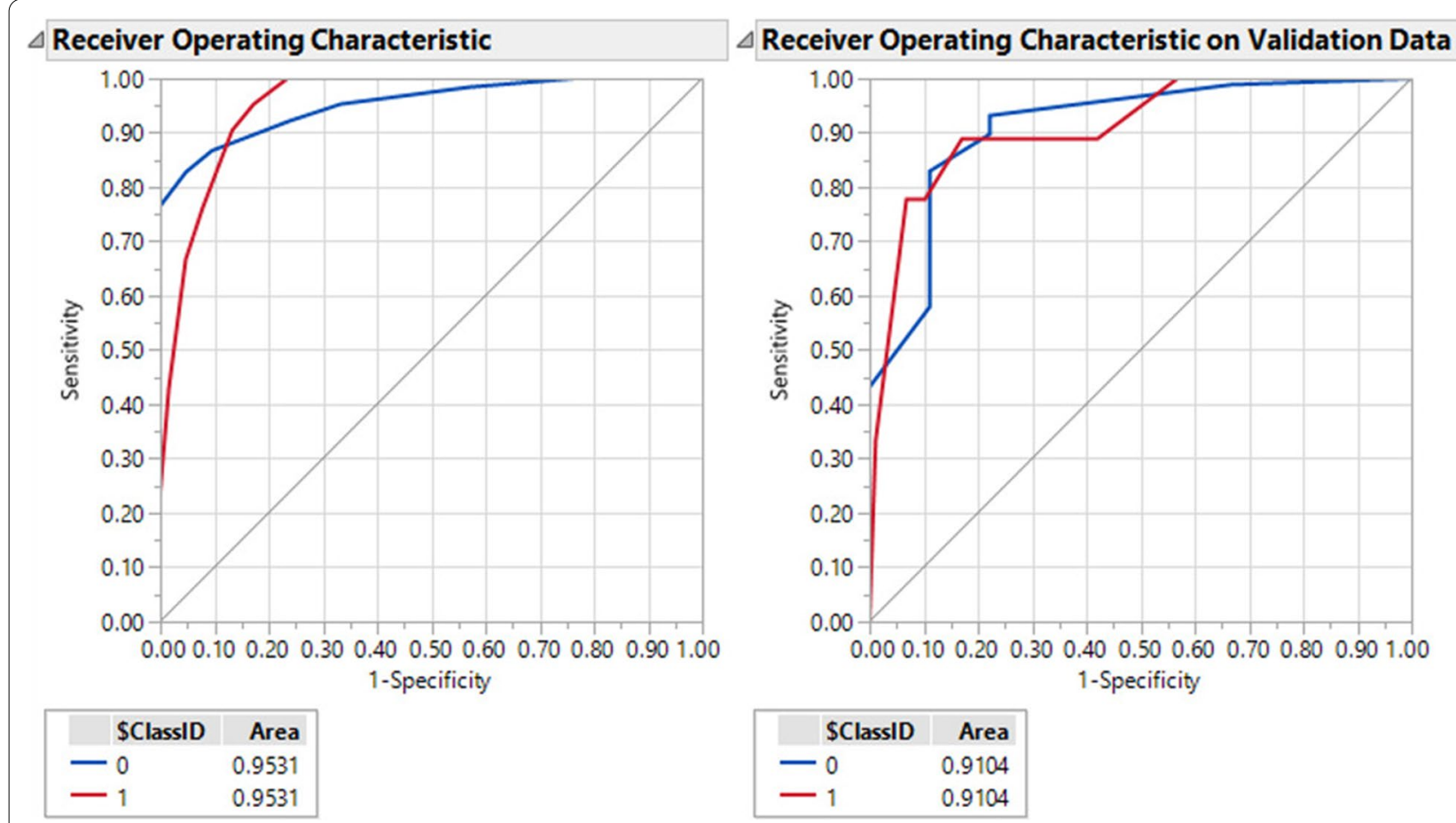

Fig. 2 AUC for the separation of hospital mortality and survivors from COVID-19

\section{Identification of high-risk patients with COVID-19}

Further investigations using PCA and LCA showed that patients with COVID-19 can be clustered to identify the high-risk patients (Fig. 4) based on the clinical data.

LCA was performed using most differentiating clinical variables obtained by SIMPLS prediction models. LCAbased clustering revealed three main clusters among the patients with COVID-19 cohorts (survivors and nonsurvivors). LCA-based clustering revealed that cluster 3 and cluster 2 had a $38 \%$ and $12.5 \%$ mortality rate. Cluster 1 was with the lowest rate of mortality $(0-1.3 \%)$ compared to clusters 2 and 3 . All 3 clusters were well depicted through a PCA plot that can verify the clustering using two unsupervised methods. Table 4 shows that although variables had different contributions to each cluster, several variables markedly impact clustering. Hence, age $<65$, lack of hypertension, lack of diabetes, alcohol consumption, and headache were highly correlated with cluster 1 and with a lower rate of mortality. On the other hand, age $>65$, nursing home, AMS, stroke, atrial fibrillation, CAD, and dementia were the most important variables correlated with cluster 3 ; chest pain and dyspnea were the most important variables correlated with cluster 2 . Also, hypertension, yno2, consolidation, $\mathrm{O} 2$ saturation $<88$, and diabetes were variables that had a similarly high probability for clusters 2 and 3 . This result showed that nursing home, dementia,
O2 saturation $<88$, diabetes, hypertension, age $>65$ are risk factors for COVID-19 survivors in clusters 2 and 3. Table 4 shows the probability of all 18 variables for each cluster in the analysis. Multivariate correlation analysis of 19 most differentiating clinical and comorbidities predictor was obtained by SIMPLS. The correlation values $>0.2$ are in red with highlighted cells (Table 5).

Further analysis showed that three clusters are separated from each other using a very good predictive $\left(Q^{2}=0.69\right)$ with high variability $\left(R^{2} Y=0.81\right)$ SIMPLSbased model using most differentiating variables (Fig. 5).

More investigations revealed that the prognosis of hospital mortality was poorly predicted using paraclinical data such as blood cell characteristics (i.e., numbers of leukocytes, neutrophils, lymphocytes, eosinophils, hemoglobin) and biochemical measures (i.e., BUN, creatine, sodium, CRP, procalcitonin [PCT], lactate, etc.) compared to clinical data and comorbidities.

\section{Discussion}

In the current study, machine learning algorithms were applied to predict hospital mortality using a prediction model based on the demographic, clinical predictors, comorbidities, and biochemical markers of patients with COVID-19. The two-component SIMPLS-based prediction model had moderate predictive power $Q^{2}=0.24$ to predict hospital mortality. The prediction model was 


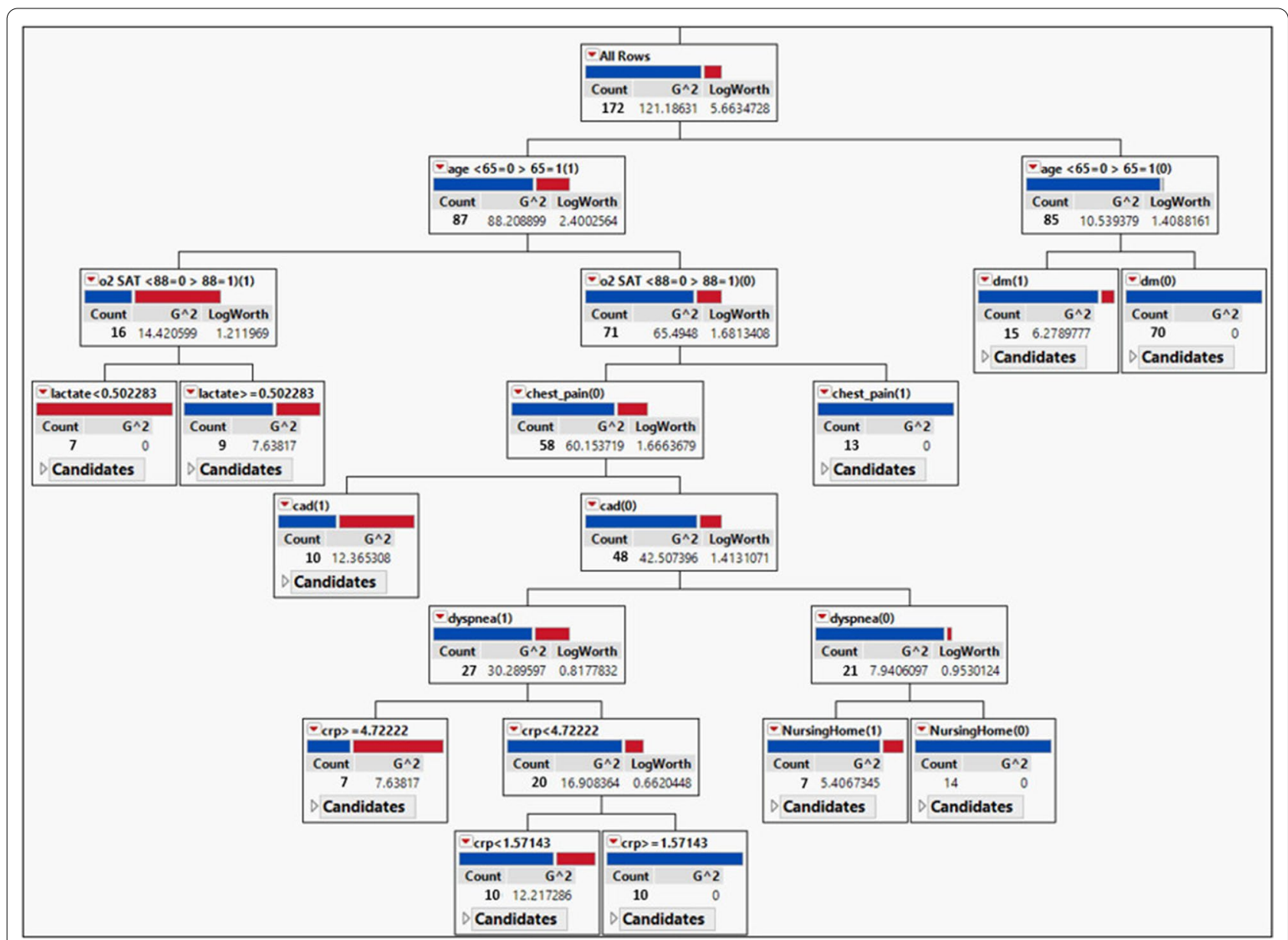

Fig. 3 Predictive partition platform analysis shows the decision tree that predicts the hospital mortality in patients with COVID-19 from survivors. Blue square: survivors, red square: hospital mortality

associated with high accuracy (AUC score of 0.91-0.95) using training and validation sets of the patient cohort. The prediction model was developed based on the 18 clinical and comorbidities, and 3 paraclinical biochemical markers uncovering most differentiating predictors that some have not been recognized through conventional statistical methods. Hence, CAD showed the highest predictive importance for in-hospital death, followed by diabetes, age $>65$, Altered Mental Status, dementia, and O2 saturation $<88 \%$. Also, LCA clustering was successful to identify high- and low-risk clusters in COVID-19 survivors. The clusters were discriminated against based on the high predictive power model $Q^{2}=0.69$. Age $<65$, lack of hypertension, and lack of diabetes were highly correlated with a lower rate of mortality among survivors while residing in the nursing home, age $>65$, AMS, stroke, atrial fibrillation, CAD, and dementia were risk factors for in-hospital mortality in COVID-19 survivors. Multivariate analysis demonstrated that there are some most differentiating predictors which are not included in the univariate method (Table 1) such as yno2, dyspnea, alcohol, $\mathrm{O} 2$ saturation, and stroke. Moreover, the multivariate analysis helped to determine the weight of the clinical predictors based on their importance in the prediction model (VIP) that is considered as the value of multivariate analysis compared to the univariate analysis. On the other hand, acute MI, CHF, O2 flow rate (lpm), Fio2, and blood pressure were significantly different between the two groups which were not selected as most differentiating predictors using SIMPLS. The combination of paraclinical data with patient demographics and comorbidities significantly improved the prediction of hospital mortality compared to when patient demographics and comorbidities or paraclinical data were independently poor predictors for the prognosis of hospital mortality. Lactate, CRP, and prothrombin were the most weighted biochemical variables that could be contributed to predicting hospital mortality.

Several other studies are published on COVID-19 mortality prediction model development. In a large cohort, 


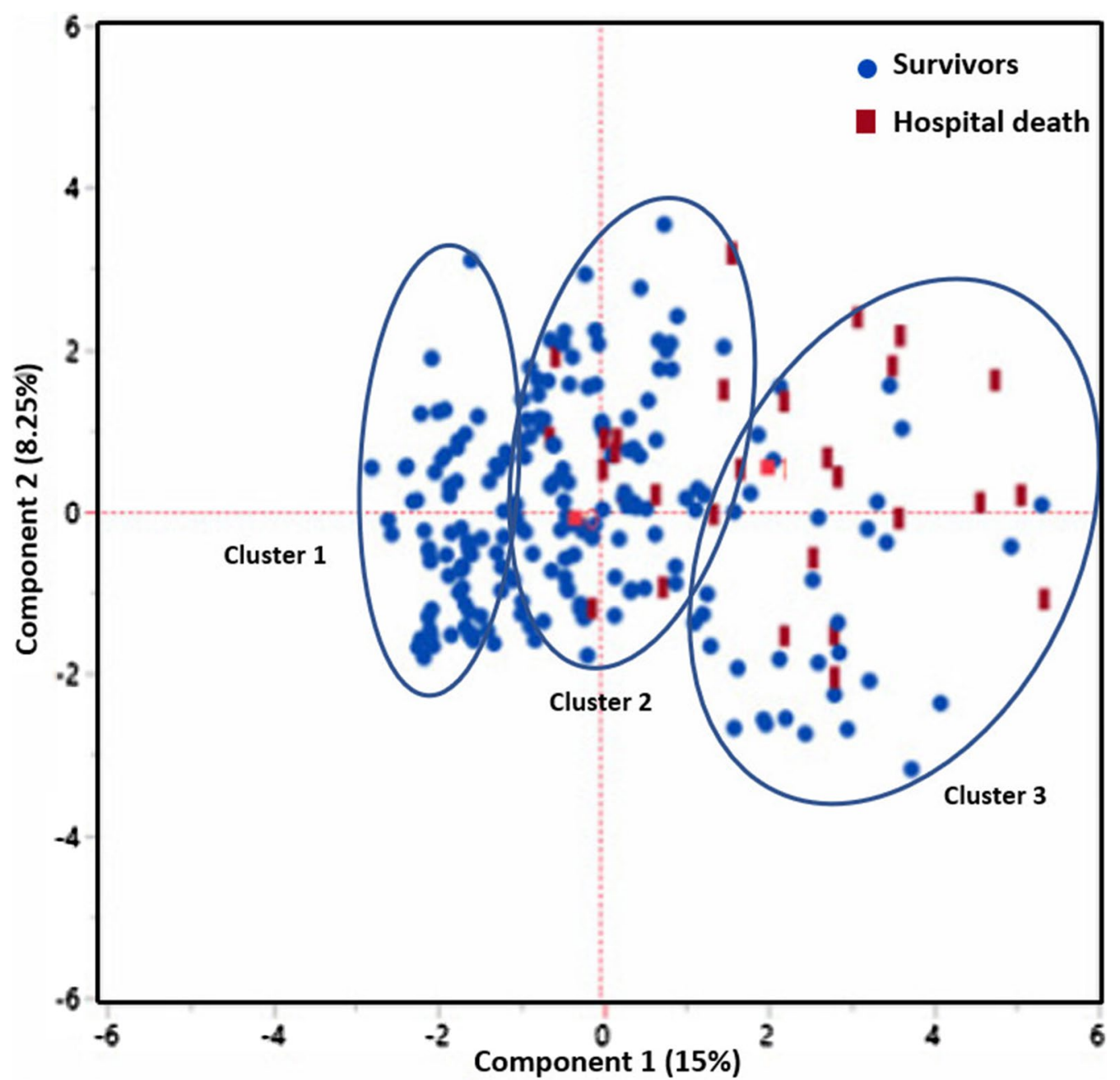

Fig. 4 PCA plot illustrates the LCA-based clustering of patients with COVID-19. Clusters 2 and 3 are associated with a higher rate of mortality. Black circle: Survivors, red square: Hospital mortality

Yadaw et al. developed a highly accurate $(\mathrm{AUC}=0.91)$ ML-based mortality prediction model, using patient's age, $\mathrm{O} 2$ saturation throughout their medical encounter, and type of patient encounter (inpatient versus outpatient and telehealth visits) [14]. Age and minimum $\mathrm{O} 2$ saturation during the encounter were the most predictive factors, which is in line with our results. Individuals aged 60 years and older represent nearly $85 \%$ of all deaths, in COVID-19 hot spots across the USA [15]. Not surprisingly, the severity of hypoxia at presentation has been extensively reported as a significant indicator of the severity of illness, specifically in acute respiratory distress syndrome, and carries strong justification to be an important predictive factor in the clinical course of COVID-19 [16, 17]. Although development and validation datasets were larger in this study, the collected data were limited to those routinely collected during hospital encounters and did not include the comprehensive list of demographics, comorbidities, biochemical tests, imaging, and omics data. Additionally, although they had large datasets, the number of dead participants was small. Knight et al. conducted a large prospective cohort, evaluating an 8-item scoring system (score range $0-21$ points) for in-hospital mortality due to COVID-19 [18]. The variables included age, gender, number of comorbidities, respiratory rate, $\mathrm{O} 2$ saturation, level of consciousness, urea level, and CRP. This scoring system revealed high discrimination for mortality (derivation cohort: AUC 0.79; validation cohort: 0.77 ); however, some potentially relevant comorbidities such as hypertension, previous myocardial infarction, and stroke were not included in data collection. Moreover, regarding the $32.2 \%$ mortality rate 
Table 4 The conditional probabilities for each cluster are shown for each response category of 20 variables in the analysis

\begin{tabular}{|c|c|c|c|c|}
\hline Variable & Category & Cluster 1 & Cluster 2 & Cluster 3 \\
\hline Age $>65$ & No & 0.8791 & 0.3844 & 0.0429 \\
\hline Age $>65$ & Yes & 0.1209 & 0.6156 & 0.9571 \\
\hline Nursing home & No & 0.9976 & 0.9509 & 0.3191 \\
\hline Nursing home & Yes & 0.0024 & 0.0491 & 0.6809 \\
\hline Smoking & No & 0.9157 & 0.9411 & 0.9255 \\
\hline Smoking & Yes & 0.0843 & 0.0589 & 0.0745 \\
\hline Alcohol & No & 0.5509 & 0.7871 & 0.9453 \\
\hline Alcohol & Yes & 0.4491 & 0.2129 & 0.0547 \\
\hline Chest pain & No & 0.8947 & 0.7753 & 0.996 \\
\hline Chest pain & Yes & 0.1053 & 0.2247 & 0.004 \\
\hline Dyspnea & No & 0.4492 & 0.2709 & 0.5011 \\
\hline Dyspnea & Yes & 0.5508 & 0.7291 & 0.4989 \\
\hline Headache & No & 0.8459 & 0.9365 & 0.9793 \\
\hline Headache & Yes & 0.1541 & 0.0635 & 0.0207 \\
\hline AMS & No & 0.9954 & 0.9214 & 0.5785 \\
\hline AMS & Yes & 0.0046 & 0.0786 & 0.4215 \\
\hline Consolidation & No & 0.8887 & 0.7795 & 0.727 \\
\hline Consolidation & Yes & 0.1113 & 0.2205 & 0.273 \\
\hline O2 saturation $<88$ & No & 0.9979 & 0.8809 & 0.855 \\
\hline O2 saturation < 88 & Yes & 0.0021 & 0.1191 & 0.145 \\
\hline yno2 & No & 0.8128 & 0.5695 & 0.5008 \\
\hline yno2 & Yes & 0.1872 & 0.4305 & 0.4992 \\
\hline CAD & No & 0.9978 & 0.9125 & 0.6985 \\
\hline CAD & Yes & 0.0022 & 0.0875 & 0.3015 \\
\hline Atrial fibrillation & No & 0.9911 & 0.9019 & 0.7769 \\
\hline Atrial fibrillation & Yes & 0.0089 & 0.0981 & 0.2231 \\
\hline Hypertension & No & 0.8206 & 0.173 & 0.1622 \\
\hline Hypertension & Yes & 0.1794 & 0.827 & 0.8378 \\
\hline PVD & No & 0.9977 & 0.9447 & 0.8524 \\
\hline PVD & Yes & 0.0023 & 0.0553 & 0.1476 \\
\hline Stroke & No & 0.9892 & 0.976 & 0.6911 \\
\hline Stroke & Yes & 0.0108 & 0.024 & 0.3089 \\
\hline Dementia & No & 0.9981 & 0.9984 & 0.4778 \\
\hline Dementia & Yes & 0.0019 & 0.0016 & 0.5222 \\
\hline Diabetes & No & 0.9772 & 0.5565 & 0.4624 \\
\hline Diabetes & Yes & 0.0228 & 0.4435 & 0.5376 \\
\hline
\end{tabular}

No and yes values are considered as the absence and presence, respectively, for the clinical variables

and elderly patient population (median age of 73 years old), this model could function differently in younger patients and/or populations at lower risk of death.

LASSO and multivariate data analysis-based prediction models showed that higher age, coronary heart disease (CHD), percentage of lymphocytes (LYM\%), procalcitonin (PCT), urea, CRP, and D-dimer (DD) could be potential risk factors for mortality of COVID. These variables could classify the COVID patients into low- and high-risk groups using a good prediction model $(\mathrm{AUC}=0.91)[19]$.

Considerable heterogenicity exists among COVID-19 mortality prediction models. Unlike our results which showed paraclinical and biochemical data have limited predictive value, in the model developed by Zhao et al. (AUC 0.83), lactate dehydrogenase and procalcitonin were among the top mortality prediction factors [20], and the COVID-AID study showed that renal failure at presentation (defined by creatinine $>2 \mathrm{mg} / \mathrm{dL}$ ), regardless of chronicity has a high impact on in-hospital mortality in hospitalized COVID-19 patients [21]. Recent studies have reported that prothrombin and CRP are associated with COIVD severity and mortality [22, 23]. In this study, we showed the correlation of decreased $\mathrm{O} 2$ and increased lactate that may indicate the higher level of the anaerobic metabolism [24] in patients with COVID-19 that are associated with mortality.

Late April 2020, a systematic review and meta-analysis showed a significantly higher rate of hypertension, diabetes, cardiovascular disease, and respiratory disease in critically ill COVID patients compared to non-critical patients [25]. Then, another systematic review and metaanalysis on risk for predicting mortality of COVID 19 patients demonstrated that dyspnea, chest tightness, hemoptysis, expectoration, and fatigue were the most significant clinical variables in association with increased risk of COVID-19 mortality. This study also showed significant increased leukocyte count and decreased lymphocyte count in non-survivors [26]. ML was successfully applied to determine COVID-19 severity by predicting the need for ICU $(\mathrm{AUC}=0.80)$ and the need for mechanical ventilation ( $\mathrm{AUC}=0.82)$ [27]. Random forest analysis showed that PCT, DD, CRP, respiratory rate, SpO2, albumin, AST/SGOT, calcium, influenza-like symptoms, and ALT/SGPT are the most important variables to predict the need for ICU. Also, CRP, DD, PCT, SpO2, respiratory rate, creatinine, total protein, albumin, calcium, and age were the most important variables to predict the need for mechanical ventilation [27]. In a similar study, $\mathrm{SpO} 2 / \mathrm{FiO} 2, \mathrm{CRP}$, estimated glomerular filtration rate (eGFR), age, Charlson score, lymphocyte count, and PCT were the most important variables for the prediction COVID severity [28]. LASSO-based prediction model showed that lymphocyte percentage, lactic dehydrogenase (LDH), neutrophil count, and DD in combination with four quantitative CT findings including pneumonia percentage in the lateral basal segment of left lower lung, the volume of the whole lung with the density of -300 to $-200 \mathrm{HU}$, pneumonia volume in both lungs and pneumonia volume in the right lung can be most important variables to prognosticate critical illness risk in hospitalized patients with COVID-19 pneumonia [29]. Age, PCT, 
Table 5 Multivariate correlation analysis of 19 most differentiating clinical and comorbidities predictor obtained by SIMPLS



The correlation values $>0.2$ are in red with highlighted cells

CRP, LDH, DD, and lymphocytes were top mortality predictors and PCT, LDH, CRP, O2 saturation, temperature, and ferritin were important predictors for the ICU need with AUC $89 \%$ and $79 \%$, respectively, in a cohort from New York [30].

Leon et al. applied the ML approach to cluster the patients with COVID into 3 groups including higher, moderate, and low rate of mortality. This study showed that the higher and lower AST, ALT, LDH, CRP, and number of neutrophils were associated with a higher and lower rate of mortality, respectively [31]. The percentages of monocytes and lymphocytes were negatively correlated with mortality [31]. Unlike our results, Leon's study showed that age, sex, and comorbidities did not contribute to the above clustering model [31].

The strengths of our study include assessing a comprehensive list of demographic, clinical, and paraclinical variables, at all stages of hospitalization (admission, during hospital stay, and hospital discharge), development of an internally validated accurately discriminating in-hospital mortality prediction model, identification of high-risk and low-risk clusters of COVID patients whose healthcare needs are different, and enrollment of PCR-proven cases of SARS-CoV2, rather than possible COVID-19 patients. SIMPLS is considered a suitable multivariate method to investigate big and complex datasets that have a relatively small sample size and many variables [32]. External validation using an external cohort may help the results to be more practicable and achievable at any time with any cohorts. Current findings in this study may improve the precise prognostication of COVID-19 mortality, classification of low and high risk, and identification of potential risk factors.

Our study has a few limitations. First, this is a singlecenter retrospective study, which might impact the data quality and generalizability. Second, although we had an acceptable sample size, the subset of dead individuals was small $(n=31)$. A major reason for this concern is that the number of predictor parameters considered by ML approaches usually exceeds that for regression, even when the same set of predictors is applied, especially since multiple interaction terms are constantly examined and continuous predictors are routinely classified. Therefore, ML methodologies require "big data" to ensure their developed models have minimized overfitting and for their potential advantages (i.e., dealing with highly nonlinear relations and complex interactions) to reach fruition.

\section{Conclusion}

In conclusion, we presented an accurate ML-based inhospital mortality prediction model for COVID-19, which can aid in clinical decision making and resource allocation. This model needs to be externally validated in larger populations and multicenter settings. 




Fig. 5 SIMPLS-based scatter plot shows a very good separation between three clusters obtained by LCA. Clusters 1 includes the patients with a lower risk of dying, and clusters 2 and 3 include patients with a higher risk of dying

\section{Abbreviations}

COVID-19: Corona Virus Disease 2019; SIMPLS: Statistically Inspired Modification of Partial Least Square; LCA: Latent Class Analysis; PCA: Principal Component Analysis; CAD: Coronary Artery Disease; AMS: Altered Mental Status; ARDS: Acute Respiratory Distress Syndrome; ML: Machine Learning; Al: Artificial Intelligence; PCR: Polymerase Chain Reaction; GCS: Glascow Coma Scale; RR: Respiratory Rate; ACE: Angiotensin Converting Enzyme; COPD:

Chronic Obstructive Pulmonary Disease; PE: Pulmonary Emboli; ILD: Interstitial Lung Disease; CHF: Congestive Heart Failure; AMI: Acute Myocardial Infarction; Afib: Atrial Fibrillation; CRF: Chronic Renal Failure; AUC: Area Under Curve; CRP: C-reactive Protein; PCT: Procalcitonin; DD: D-dimer.

\section{Acknowledgements}

Not applicable.

\section{Authors' contributions}

MB had contributed to methodology, software, formal analysis, and writing. RD was involved in writing, original draft, review, editing, and formatting. AV designed the study and interpreted the data. MM took part in conceptualization, methodology, supervision, and investigation. All authors read and approved the final manuscript.

\section{Funding}

This research did not receive any specific grant from funding agencies in the public, commercial, or not-for-profit sectors.

Availability of data and materials

The datasets used and/or analyzed during the current study are available from the corresponding author on reasonable request.

\section{Declarations}

Ethics approval and consent to participate

Informed consent was waived due to the nature of study being retrospective.

Consent for publication

Not applicable.

\section{Competing interests}

The authors declare they have no conflict of interest. 


\begin{abstract}
Author details
${ }^{1}$ Department of Critical Care Medicine, University of Calgary, Alberta, Canada. ${ }^{2}$ Department of Biological Science, University of Calgary, Alberta, Canada. ${ }^{3}$ Division of Pulmonary and Critical Care Medicine, Johns Hopkins University, Baltimore, MD 21218, USA. ${ }^{4}$ Division of Pulmonary and Critical Care, Miami VA Medical Center, Miami, FL, USA. ${ }^{5}$ Division of Pulmonary and Critical Care, Department of Medicine, University of Miami, Miami, FL, USA.
\end{abstract}

Received: 18 July 2021 Accepted: 27 August 2021

Published online: 08 September 2021

\section{References}

1. Dhama K, Khan S, Tiwari R, Sircar S, Bhat S, MalikYS, Singh KP, Chaicumpa W, Bonilla-Aldana DK, Rodriguez-Morales AJ. Coronavirus disease 2019COVID-19. Clin Microbiol Rev. 2020;33(4):e00028-e120.

2. Hassan SA, Sheikh FN, Jamal S, Ezeh JK, Akhtar A. Coronavirus (COVID19): a review of clinical features, diagnosis, and treatment. Cureus. 2020;12(3):e7355.

3. Guan WJ, Ni ZY, Hu Y, Liang WH, Ou CQ, He JX, Liu L, Shan H, Lei CL, Hui DSC, et al. Clinical characteristics of coronavirus disease 2019 in China. N Engl J Med. 2020;382(18):1708-20.

4. Chen Y, Ouyang L, Bao FS, Li Q, Han L, Zhang H, Zhu B, Ge Y, Robinson P, $\mathrm{Xu} \mathrm{M}$, et al. A multimodality machine learning approach to differentiate severe and nonsevere COVID-19: model development and validation. J Med Internet Res. 2021;23(4):e23948.

5. Elwazir MY, Hosny S. Artificial intelligence in COVID-19 ultrastructure. J Microsc Ultrastruct. 2020;8(4):146-7.

6. Chou EH, Wang CH, Hsieh YL, Namazi B, Wolfshohl J, Bhakta T, Tsai CL, Lien WC, Sankaranarayanan G, Lee CC, et al. Clinical features of emergency department patients from early COVID-19 pandemic that predict SARS-CoV-2 infection: machine-learning approach. West J Emerg Med. 2021;22(2):244-51.

7. Venturini S, Orso D, Cugini F, Crapis M, Fossati S, Callegari A, Pellis T, Tonizzo M, Grembiale A, Rosso A, et al. Classification and analysis of outcome predictors in non-critically ill COVID-19 patients. Intern Med J. 2021;51(4):506-14.

8. Boulesteix AL, Strimmer K. Partial least squares: a versatile tool for the analysis of high-dimensional genomic data. Brief Bioinform. 2007;8(1):32-44.

9. de Jong S. SIMPLS: an alternative approach to partial least squares regression. Chemom Intell Lab Syst. 1993;18(3):251-63.

10. Wold S, Sjöström M, Eriksson L. PLS-regression: a basic tool of chemometrics. Chemom Intell Lab Syst. 2001;58(2):109-30.

11. Eriksson L, Johansson E, Kettaneh-Wold NTJ, Wikstrom C, Wold S. Multiand megavariate data analysis basic principles and applications (part I), chapter 4. In: Umetrics; 2006.

12. Peng DX, Lai F. Using partial least squares in operations management research: a practical guideline and summary of past research. J Oper Manag. 2012;30(6):467-80.

13. Wu J-F, Wang Y. Multivariate analysis of metabolomics data. In: Qi X, Chen X, Wang Y, editors. Plant metabolomics: methods and applications. Dordrecht: Springer; 2015. p. 105-22.

14. Yadaw AS, Li YC, Bose S, lyengar R, Bunyavanich S, Pandey G. Clinical features of COVID-19 mortality: development and validation of a clinical prediction model. Lancet Digit Health. 2020;2(10):e516-25.

15. Bhatraju PK, Ghassemieh BJ, Nichols M, Kim R, Jerome KR, Nalla AK, Greninger AL, Pipavath S, Wurfel MM, Evans L, et al. Covid-19 in critically ill patients in the Seattle region—case series. N Engl J Med. 2020;382(21):2012-22

16. Duca A, Piva S, Focà E, Latronico N, Rizzi M. Calculated decisions: BresciaCOVID respiratory severity scale (BCRSS)/algorithm. Emerg Med Pract. 2020;22(5 Suppl):Cd1-2

17. Grasselli G, Zangrillo A, Zanella A, Antonelli M, Cabrini L, Castelli A, Cereda D, Coluccello A, Foti G, Fumagalli R, et al. Baseline characteristics and outcomes of 1591 patients infected with SARS-CoV-2 admitted to ICUs of the Lombardy Region, Italy. JAMA. 2020;323(16):1574-81.

18. Knight SR, Ho A, Pius R, Buchan I, Carson G, Drake TM, Dunning J, Fairfield CJ, Gamble C, Green CA, et al. Risk stratification of patients admitted to hospital with covid-19 using the ISARIC WHO Clinical Characterisation Protocol: development and validation of the 4C Mortality Score. BMJ. 2020;370:m3339.

19. Shang Y, Liu T, Wei Y, Li J, Shao L, Liu M, Zhang Y, Zhao Z, Xu H, Peng Z, et al. Scoring systems for predicting mortality for severe patients with COVID-19. EClinicalMedicine. 2020;24:100426.

20. Zhao Z, Chen A, Hou W, Graham JM, Li H, Richman PS, Thode HC, Singer AJ, Duong TQ. Prediction model and risk scores of ICU admission and mortality in COVID-19. PLoS ONE. 2020;15(7):e0236618.

21. Hajifathalian K, Sharaiha RZ, Kumar S, Krisko T, Skaf D, Ang B, Redd WD, Zhou JC, Hathorn KE, MCCarty TR, et al. Development and external validation of a prediction risk model for short-term mortality among hospitalized U.S. COVID-19 patients: a proposal for the COVID-AID risk tool. PLoS ONE. 2020;15(9):e0239536.

22. von Meijenfeldt FA, Havervall S, Adelmeijer J, Lundström A, Rudberg AS, Magnusson M, Mackman N, Thalin C, Lisman T. Prothrombotic changes in patients with COVID-19 are associated with disease severity and mortality. Res Pract Thromb Haemost. 2021;5(1):132-41.

23. Bannaga AS, Tabuso M, Farrugia A, Chandrapalan S, Somal K, Lim VK, Mohamed S, Nia GJ, Mannath J, Wong JL, et al. C-reactive protein and albumin association with mortality of hospitalised SARS-CoV-2 patients: a tertiary hospital experience. Clin Med (Lond). 2020;20(5):463-7.

24. Li Z, Liu G, Wang L, Liang Y, Zhou Q, Wu F, Yao J, Chen B. From the insight of glucose metabolism disorder: oxygen therapy and blood glucose monitoring are crucial for quarantined COVID-19 patients. Ecotoxicol Environ Saf. 2020;197:110614-110614.

25. Zheng Z, Peng F, Xu B, Zhao J, Liu H, Peng J, Li Q, Jiang C, Zhou Y, Liu S, et al. Risk factors of critical \& mortal COVID-19 cases: A systematic literature review and meta-analysis. J Infect. 2020;81(2):e16-25.

26. Yang L, Jin J, Luo W, Gan Y, Chen B, Li W. Risk factors for predicting mortality of COVID-19 patients: a systematic review and meta-analysis. PLoS ONE. 2020;15(11):e0243124

27. Patel D, Kher V, Desai B, Lei X, Cen S, Nanda N, Gholamrezanezhad A, Duddalwar $\vee$, Varghese B, Oberai AA. Machine learning based predictors for COVID-19 disease severity. Sci Rep. 2021;11(1):4673.

28. Marcos M, Belhassen-García M, Sánchez-Puente A, Sampedro-Gomez J, Azibeiro R, Dorado-Díaz PI, Marcano-Millán E, García-Vidal C, MoreiroBarroso MT, Cubino-Bóveda N, et al. Development of a severity of disease score and classification model by machine learning for hospitalized COVID-19 patients. PLOS ONE. 2021;16(4):e0240200.

29. Liu Q, Pang B, Li H, Zhang B, Liu Y, Lai L, Le W, Li J, Xia T, Zhang X, et al. Machine learning models for predicting critical illness risk in hospitalized patients with COVID-19 pneumonia. J Thorac Dis. 2021;13(2):1215-29.

30. Hou W, Zhao Z, Chen A, Li H, Duong TQ. Machining learning predicts the need for escalated care and mortality in COVID-19 patients from clinical variables. Int J Med Sci. 2021;18(8):1739-45.

31. Benito-León J, Del Castillo MD, Estirado A, Ghosh R, Dubey S, Serrano J. Using unsupervised machine learning to identify age- and sex-independent severity subgroups among COVID-19 patients in the emergency department. J Med Internet Res. 2021;23:e25988.

32. Eriksson L, Antti H, Gottfries J, Holmes E, Johansson E, Lindgren F, Long I, Lundstedt T, Trygg J, Wold S. Using chemometrics for navigating in the large data sets of genomics, proteomics, and metabonomics (gpm). Anal Bioanal Chem. 2004;380(3):419-29.

\section{Publisher's Note}

Springer Nature remains neutral with regard to jurisdictional claims in published maps and institutional affiliations. 